\title{
ACTIVIDAD NEUROPROTECTORA DE Solanum ovalifolium (SOLANACEAE) CONTRA LA TOXICIDAD INDUCIDA POR ROTENONA EN Drosophila melanogaster
}

\section{NEUROPROTECTIVE ACTIVITY OF Solanum ovalifolium (SOLANACEAE) AGAINST THE TOXICITY INDUCED BY ROTENONE IN Drosophila melanogaster}

\section{Erika V. JIMÉNEZ', Jennifer TOVAR', Oscar M. MOSQUERA*, Fernando CARDOZO $^{2}$}

Grupo de Biotecnología-Productos Naturales, Escuela de Tecnología Química Universidad Tecnológica de Pereira. Carrera 27 No 10-02 Barrio Los Álamos. PereiraColombia

2 Departamento de Ciencias Biomédicas y Farmacéuticas, Universidad de Montana, Missoula, Montana-USA.

*Autor Corresponsal. E-mail: omosquer@utp.edu.co

\author{
Historia del Artículo \\ Recibido: Julio 31, 2016 \\ Evaluado: Diciembre 19, 2016 \\ Aceptado: Enero 21, 2017 \\ Disponible: Febrero 07, 2017
}

Resumen |

Los objetivos de este trabajo fueron evidenciar la presencia de flavonoides en el extracto metanólico de Solanum ovalifolium por cromatografía líquida de alta eficiencia (HPLC-UV), analizar la actividad antioxidante por los métodos de 1,1-difenil-2-picrilhidrazilo $\left(\mathrm{DPPH}^{\bullet}\right.$ ) y ácido 2,2'-azinobis-(3-etilbenzotiazolina)-6-sulfónico $\left(\mathrm{ABTS}^{*+}\right)$, cuantificar el glutatión (GSH), las unidades de enzima antioxidante superóxido dismutasa (SOD) y determinar el efecto neuroprotector contra la toxicidad inducida por rotenona (100 $\mu \mathrm{M})$ con el modelo in vivo de Drosophila melanogaster mediante geotaxis negativa y cuantificación de la dopamina (DA) de las células cerebrales por (HPLC-UV). A través del perfil fitoquímico por cromatografía líquida de alta eficiencia (HPLC-UV) se evidenció un alto contenido de flavonoides, principalmente dihidroxiflavona (83.33\%) y flavona-flavonol (16.66\%), el extracto presentó una concentración media inhibitoria para los radicales $\mathrm{DPPH}^{\circ}$ y $\mathrm{ABTS}^{\circ+}$ de $179.8 \mu \mathrm{g} / \mathrm{mL}$ y $34.4 \mu \mathrm{g} / \mathrm{mL}$ respectivamente. En el extracto de S. ovalifolium a $1000 \mathrm{mg} / \mathrm{L}$ se cuantificó $4.28 \mathrm{nmol}$ de $\mathrm{GSH} / \mathrm{mL}$ de extracto y 1.43 unidades SOD/mL del extracto. En la evaluación del efecto neuroprotector, las moscas macho variedad silvestre (75) co-expuestas a 7 días de tratamiento con el extracto metanólico de S. ovalifolium $0.1 \%$ presentaron un menor efecto tóxico y una protección completa contra el estrés oxidativo inducido por rotenona. El contenido de dopamina fue de $61-65 \mu \mathrm{g} / \mathrm{L}$. Adicionalmente, este trabajo confirma la utilidad del modelo in vivo de $D$. melanogaster como una etapa en la evaluación de posibles medicamentos neuroprotectores.

Palabras Clave: Antioxidante, enfermedad de Parkinson, estrés oxidativo, flavonoides, neuroprotección.

\section{Abstract}

The aims of this work were study the flavonoids from methanol Solanum ovalifolium extract by High Performance Chromatography (HPLC-UV), analyze the antioxidant activity by the free radical method 2,2-diphenyl -1-picrylhydrazyl hydrate (DPPH') and the 2,2'azinobis-(3-ethylbenzothiazoline-6-sulfonic acid $\left(\mathrm{ABTS}^{*+}\right)$, quantify glutathione (GSH) and antioxidant enzyme superoxide dismutase (SOD) of S. ovalifolium extract and in vitro neuroprotective effect against toxicity induced by rotenone in Drosophila melanogaster in the negative geotaxis assay and dopamine extracted from brain cell and analyzed by (HPLC-UV). The phytochemical profile by HPLC-UV showed high content of dihydoxyflanvone (83.33\%) and flavones-flavonol (16.66\%), the extract exhibited a halfmaximal inhibitory concentration $179.8 \mu \mathrm{g} / \mathrm{mL}$ for $\mathrm{DPPH}^{\circ}$. and $34.4 \mu \mathrm{g} / \mathrm{mL}$ for ABTS ${ }^{\circ+}$. In the S. ovalifolium extract was quantified $4.28 \mathrm{nmol} \mathrm{GSH} / \mathrm{mL}$ of extract and 1.43 units of SOD/mL of extract. Adult male wild-type flies (75) were coexposed to rotenone $(100 \mu \mathrm{M})$ and S. ovalifolium methanol extract (0.1\%) during 7 days. S ovalifolium treatment offered total protection against rotenone effects, the flies exposed had better performance in the negative geotaxis assay and the dopamine concentration by HPLC-UV was 61-65 $\mathrm{\mu g} / \mathrm{L}$. In addition, this study confirms the utility of this model to investigate new potential neuroprotective drugs.

Keywords: Antioxidant, Parkinson's disease, oxidative stress, flavonoids, neuroprotection. 


\section{INTRODUCCIÓN |}

El cerebro es sensible al daño oxidativo debido a su elevada utilización de oxígeno, alto contenido de ácidos grasos poli insaturados oxidables, y la presencia de metales electrónicamente activos. El estrés oxidativo aumenta con la edad y por lo tanto se puede considerar como un factor causativo importante en varias enfermedades neurodegenerativas, como lo es la enfermedad de Parkinson (EP) (González et al., 2016).

La enfermedad de Parkinson (EP) es un trastorno neurodegenerativo que genera rigidez, pérdida del control motriz e inestabilidad postural, la lesión fundamental de la EP es la pérdida de neuronas dopamirégicas localizadas en la parte compacta de la sustancia negra (SN) que está en los ganglios basales y la formación de inclusiones filamentosas interneuronales citoplasmáticas ricas en $\alpha$-sinucleina (cuerpos de lewy) (Bosson et al., 2015).

Con el aumento de la expectativa de vida en países desarrollados, crece la necesidad de prevenir las enfermedades asociadas edad, prestando interés en el efecto neuroprotector de antioxidantes naturales, siendo las plantas una fuente importante de compuestos con estas características (Mosquera et al., 2007; Sabir y Rocha, 2008; Samarth et al., 2008).

Para el estudio de este tipo de sustancias se han implementado modelos in vivo, uno de ellos es Drosophila melanogaster, que expresa la a-sinucleina humana en la región cerebral, por lo que este modelo ofrece muchas ventajas para el estudio celular y molecular de la patologia de la enfermedad, entre ellas el alto grado de conservacion entre las vias fundamentales biologicas del genoma humano y el de Drosophila (Rubin et al., 2000), la reducción del tiempo en la obtención de resultados debido alcorto ciclo de vida de la mosca, el gran numero de progenia, la disponibilidad de tecnicas y herramientas par al manipulación genética y el conocimiento detallado de la anatomia y fenotipo de la misma.

La rotenona es un pesticida natural derivado de las raíces de especies del género Lonchocarpus. Es un inhibidor específico de NADH deshidrogenasa (complejo I) y debido a su alta liposolubilidad, puede cruzar la barrera hematoencefálica rápidamente, acumulándose en organelos subcelulares como la mitocondria, afectando la fosforilación oxidativa (Uversky, 2004). En el modelo de estudio se ha demostrado la perdida selectiva de neuronas dopaminergicas y disfuncion locomotora severa en las moscas que fueron expuestas a rotenona (Coulom y Birman, 2004).

En los organismos vivos las enzimas actúan como mecanismos de defensa frente al estrés oxidativo, ejemplos de estas son el súper óxido dismutasa (SOD), que inactiva radicales de oxígeno y la glutationa (GSH) que ayuda en la eliminación de xenobióticos y defensa a las células ante el estrés oxidativo.

De esta manera se pueden evaluar sustancias o agentes externos con acción antioxidante y neuroprotectora, como los flavonoides que han sido ampliamente estudiados por tener la capacidad de inhibir procesos oxidativos en condiciones in vitro e in vivo, ya sea estimulando la acción de las enzimas mencionadas o mimetizando su comportamiento. Investigaciones sugieren que los flavonoides pueden ser capaces de contrarrestar el daño neuronal subyacente a estos trastornos (Diniz et al., 2015).

Solanum ovalifolium (Solanaceae) es una especie arbórea de hasta $8 \mathrm{~m}$ de altura y $20 \mathrm{~cm}$ de diámetro, de hojas ovaladas a lanceoladas pubescentes, inflorescencia paniculada, flores de menos de $2 \mathrm{~cm}$ de longitud, de color blanco a lila. Esta familia ha presentado importantes actividades in vitro antibacterial, antifúngica, antioxidante y citotóxica (J Niño et al., 2006), anti protozoaria (Londoño et al., 2016), además la riqueza química de las especies Solanum en asteroides, saponinas, antraquinonas, compuestos fenólicos, alcaloides y destacada presencia de flavonoides revela la importancia de evaluar actividades biológicas de ésta especie, como las actividades antioxidante y neuroprotectora (Correa et al., 2011; Londoño et al., 2016; J Niño et al., 2006; Niño Osorio et al., 2007; Ruiz-Martínez et al., 2011; Veličković et al., 2013).

Con el fin de contribuir a la exploración de nuevas alternativas antioxidantes que podrían usarse como agentes neuroprotectores, este estudio tuvo como objetivos evaluar el efecto neuroprotector del extracto metanólico de la especie Solanum ovalifolium (Solanaceae) frente a la toxicidad inducida por la rotenona en $D$. melanogaster, la actividad antioxidante in vitro por los métodos (DPPH ${ }^{\circ}$ ) y $\left(\mathrm{ABTS}^{*+}\right)$, cuantificación de tioles libres, y superóxido dismutasa.

\section{MATERIALES Y MÉTODOS}

\section{Materiales}

En HPLC se utilizaron solventes (Metanol, Acetonitrilo, Isopropanol, Hexano) grado HPLC (Mallinckrodt, Kansas City, Missouri, USA), estándar de Clorhidrato de Dopamina (Merk, Darmstadt, Alemania) y para toxicidad inducida Rotenona (Sigma-Aldrich, Saint Louis, Missouri), Hidróxido de sodio (Merk, Darmstadt, Alemania), Fosfato de potasio $\left(\mathrm{KH}_{2} \mathrm{PO}_{4}\right)$ 98\% (Carlo Erba, Milano, Italia) y Fosfato Bibásico de sodio Anhídrido 99.8\% (J.T. Baker, Phillipsburg, USA).

\section{Material Vegetal}

S. ovalifolium fue colectada en la zona de reserva natural, Bremen-La Popa, localizada en jurisdicción del municipio de Filandia (Quindío-Colombia), con las coordenadas: 4 40' 27 " N y 75 37'56" W. La especie fue identificada por el taxónomo F.J. Roldán, un voucher de ésta 
planta se encuentra en el Herbario de la Universidad de Antioquia con el número FJR 3920.

\section{Obtención de los extractos}

Se tomaron $300 \mathrm{~g}$ de hojas secas de S. ovalifolium para extracción por maceración pasiva con solventes iniciando con $n$-hexano, siguiendo con diclorometano, y por último con metanol. Los extractos se concentraron a presión reducida a $45^{\circ} \mathrm{C}$ y se almacenaron a $-10^{\circ} \mathrm{C}$ (Jaime Niño et al., 2006). El extracto empleado para los bioensayos fue aquel obtenido de la extracción con metanol, al cual se le asignó el código interno de clasificación para el Laboratorio de biotecnología y productos naturales de UTP 99.

\section{Caracterización fitoquímica}

La caracterización fitoquímica preliminar del extracto metanólico se estableció a través del perfil por cromatografía de capa delgada en cromatofolios de sílica gel $60 \mathrm{~F}_{254}$ eluídos con acetato de etilo-metanol-agua (100:13,5:10) (Wagner y Bladt, 1996), la cromatografía liquida de alta eficiencia (HPLC-DAD) se realizó en una columna LiChrospher ${ }^{\circledR} 100$ rp-18 endcapped $(5 \mu \mathrm{m})$ de $125 \mathrm{~mm}$ de longitud, eluída en gradiente de metanol-ácido fórmico $1 \%$ (0:100 v/v) hasta (100:0 v/v) con detección a 254, 280, 330, 350 y $380 \mathrm{~nm}$ en un Cromatógrafo HPLC Agilent1100 usando el software Chemstation versión B.o.3.

\section{Actividad Antioxidante in vitro}

\section{Método DPPH ${ }^{\bullet}$}

Con el método fotométrico del radical difenilpicrilhidrazilo $\left(\mathrm{DPPH}^{*}\right)$ descrito por (Brand-Williams et al., 1995) con algunas modificaciones. La capacidad captadora de electrones se determinó mediante la reacción de $100 \mu \mathrm{L}$ de una solución de DPPH ${ }^{\bullet}$ a $20 \mathrm{mg} / \mathrm{L}$ y $25 \mu \mathrm{L}$ de una solución del extracto a $1000 \mathrm{mg} / \mathrm{L}$ durante 30 minutos en condiciones de oscuridad y a temperatura ambiente, después de este periodo se realizó la medición de la absorbancia $517 \mathrm{~nm}$ ( $\left.\mathrm{A}_{\text {muestra }}\right)$. Como control negativo de utilizó el solvente en el cual habían sido solubilizados los extractos $\left(A_{\text {control(-) }}\right)$ y como control positivo hidroquinona a $1000 \mathrm{mg} / \mathrm{L}$. La reducción del radical fue medida a $517 \mathrm{~nm}$. El porcentaje actividad antioxidante $(A A)$, se determinó mediante la siguiente ecuación: $\left[\% A A=\left[\left(A_{\text {control(-) }}-A_{\text {muestra }} /\right.\right.\right.$ $\left.A_{\text {Control(-) }}\right] \times$ 100]. También se realizó una curva de calibración de hidroquinona para establecer el IC50 a las concentraciones de 5, 10, 2040 y $80 \mathrm{mg} / \mathrm{L}$.

\section{Método del ABTS $^{\cdot+}$}

La capacidad de inhibir el radical 2,2'-azino-bis-(3etilbenzotiazolina)-6-sulfonato de amonio $\left(\right.$ ABTS $^{\bullet+}$ ) del extracto estudiado se determinó siguiendo el método propuesto por (Re et al., 1999) con algunas modificaciones. El radical se generó mediante de oxidación del ABTS a 3,5 $\mathrm{mM}$ con persulfato de potasio a $1.25 \mathrm{mM}$. Para la realización del ensayo se tomaron $6 \mu \mathrm{L}$ de extracto a $1000 \mathrm{mg} / \mathrm{L}$ y se mezclaron con $294 \mu \mathrm{L}$ de la solución de $\mathrm{ABTS}^{\circ+}$ previamente preparada y ajustada a un valor de absorbancia de 0,700 \pm 0,002 con etanol. Esta mezcla se dejó reaccionar durante media hora en ausencia de luz y a temperatura ambiente. Los controles negativo y positivo fueron los mismos utilizados en el ensayo con $\mathrm{DPPH}^{\circ}$ al igual que la ecuación empleada para la determinación de la actividad antioxidante. La reducción del $\mathrm{ABTS}^{\bullet+}$ se midió a $734 \mathrm{~nm}$.

\section{Glutatión (GSH)}

El nivel de glutationa (GSH) reducida fue determinado por el método descrito por (Samarth et al., 2008) con algunas modificaciones. Los tioles libres fueron determinados mediante un lector de microplacas Fisher Scientific Multiscan Go UV/VIS 10040, en placas de 96 pozos y un volumen final de $250 \mu \mathrm{L}$. Se hicieron reaccionar $95 \mu \mathrm{L}$ del extracto vegetal $(125 \mu \mathrm{L} \mathrm{GSH}$ estándar para la curva de calibración) con $30 \mu \mathrm{L}$ de buffer fosfato de sodio ( $\mathrm{Na}_{2} \mathrm{HPO}_{4}$ 0.5M, pH 8) y $125 \mu \mathrm{L}$ DTNB (ácido 5-5'-ditiobis-(2nitrobenzoico) 10mM (reactivo de Ellman's). La mezcla fue agitada e incubada por $15 \mathrm{~min} y$ posteriormente se determinó la absorbancia a una longitud de onda de $412 \mathrm{~nm}$.

\section{Súper óxido dismutasa (SOD)}

El sistema Xantina/Xantina oxidasa fue utilizado como fuente para la generación de un flujo de anión superoxido que se encarga de la oxidación de la hidroxilamina a nitrito, cuya concentración puede ser medida debido a la generacion de un color rosa, mediante fotometria UV/visible (Yang et al., 2004). Los reactivos utilizados tanto para la curva de calibración, como para el análisis de las muestras fueron: $\alpha-$ naftilamina $7 \mathrm{mM}$, ácido Sulfanílico $19 \mathrm{mM}$, Buffer con $\mathrm{K}_{2} \mathrm{HPO}_{4}, \mathrm{KCN}$ en buffer de fosfatos $10 \mathrm{mM}$, xantina cloruro de hidroxilamina $1 \mathrm{mM}$, xantina oxidasa $0.2 \mathrm{mg}$ Prot $/ \mathrm{mL}$, SOD estándar 4.16 Unit/ $\mu \mathrm{L}$. Para la preparación de la muestra se hicieron reaccionar $150 \mu \mathrm{L}$ Buffer de fosfato, $5 \mu \mathrm{L}$ del extracto, $40 \mu \mathrm{L}$ agua desionizada, $15 \mu \mathrm{L}$ Xantina, $15 \mu \mathrm{L}$ Hidroxilaminahidroclorada, $75 \mu \mathrm{L}$ Xantina oxidasa. La mezcla se dejó incubar durante 20 min a temperatura ambiente en ausencia de luz y posteriormente se detuvo la reacción por medio de un baño de hielo, para adicionar inmediatamente después en las celdas correspondientes $100 \mu \mathrm{L}$ de Ácido y $100 \mu \mathrm{L}$ de a-naftilamina, $100 \mu \mathrm{L}$ de la muestra (o el estándar para realizar la curva de calibración) se mezcló e incubó 20 minutos a temperatura ambiente, midiendo la absorbancia a $529 \mathrm{~nm}$.

\section{Material biológico}

Las moscas D. melanogaster silvestres fueron obtenidas del laboratorio de Biología, de la Universidad Nacional de Manizales, Caldas- Colombia.

Alimentación, crianza y sincronización de la edad de $D$. melanogaster

Las moscas fueron criadas en $20 \mathrm{~mL}$ del alimento gelificado preparado con banano, levadura, micostatin y ácido propiónico en frascos de vidrio con tapones estériles. 
El ciclo de reproducción inició en la etapa embrionaria, seguido de tres estadios larvales y la etapa pupal, de donde salieron nuevas moscas alcanzando su madurez sexual en pocas horas (Hosamani y Muralidhara, 2009).

Al sincronizar la edad, se inició la separación de los machos durante ocho días a partir del segundo día de eclosión, para ello las moscas nacidas fueron introducidas en recipientes de vidrio pequeños con flujo de aire y vapores producidos por de éter etílico durante dos minutos (una exposición mayor podría causar intoxicación), rápidamente los machos fueron diferenciados de las hembras visualizando a través de un estereoscopio o lupa (Flagg, 1988). Los machos separados (9-10 días de edad) se usaron para todos los ensayos, la manipulación de las moscas y preparación de alimento se hizo en condiciones estériles en cabina de flujo laminar.

Comportamiento y actividad locomotora de Drosophila melanogaster empleando el ensayo de geotaxis negativa

La geotaxis es el movimiento de un organismo al estímulo de la gravedad, geotaxis negativa es una respuesta de escape innata de Drosophila melanogaster que consiste en la elevación de la mosca (contraria a la gravedad). Esta característica es aprovechada para medir la capacidad locomotora de las moscas como respuesta a variaciones en la alimentación. Para tal fin, se adaptó el modelo RING (Rapid Iterative Negative Geotaxis) que se muestra en la figura 1, donde fueron depositadas moscas en recipientes transparentes o tubos geotáxicos (Falcon $115 \times 30 \mathrm{~mm}$ ) $(20$ moscas/tubo), los cuales son asegurados en una caja de madera con visualización frontal e iluminación en la parte superior, el modelo RING implementado permitía analizar de 6 tubos simultáneamente(Gargano et al., 2005).

Se evaluó el efecto modulador del extracto vegetal sobre los inducidos por la rotenona; mortalidad, disfunción locomotora y neurotoxicidad de 720 moscas macho adultas expuestas a diferentes tratamientos donde: T1: extracto (0.1\%), T2: rotenona (100uM) T3: extracto + rotenona (0.1\%100uM), T4: Ácido Ascórbico (Vitamina C) (0.1\%), T5: Testigo. Se realizó un monitoreo regular para determinar la incidencia de estos tratamiento en la mortalidad y el déficit locomotor.

El experimento consistió en depositar las moscas macho previamente anestesiadas a los tubos geotáxicos que fueron asegurados en posición invertida a la caja de madera, pasado el efecto de la anestesia se procedió a golpear vigorosamente 3 veces el aparato contra la superficie de contacto e inmediatamente se inició la captura de imágenes digitales en modo ráfaga durante 10 segundos usando una cámara Samsung DV150F (Samsung, China) que se encontraba a una distancia de $30 \mathrm{~cm}$ del RING. Este procedimiento se realizó 5 veces con un tiempo de receso de 30 entre cada repetición. Para los análisis estadísticos se utilizó solo la foto correspondiente a los 6 segundos, contando el número de moscas que ascendieron una altura superior a la mitad de la longitud del tubo.

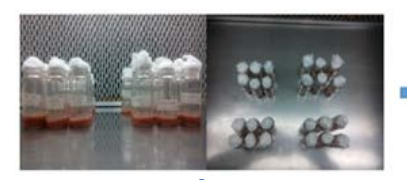

A

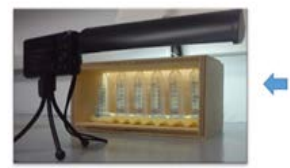

D
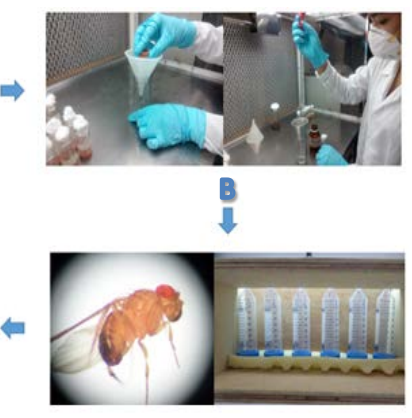

C

Figura 1. Modelo Geotaxis negativa. A) Viales con moscas macho expuestos a 7 días de tratamiento, B) Anestesia de las moscas con vapores de éter, C) Moscas introducidas en tubos geotáxicos en posición vertical, para análisis de geotaxis negativa, D) Captura digital en ráfaga de la ascensión de las moscas tras golpearla sobre la superficie. Las imágenes fueron tomadas en el proceso.

Contenido de dopamina en Drosophila melanogaster mediante análisis por HPLC-UV del efecto modulatorio de los extractos metanólicos en estudio

Preparación de la muestra:

Para evaluar el contenido de dopamina en las células neuronales, las moscas fueron anestesiadas con di-etil éter durante un minuto, posteriormente fueron congeladas a $4^{\circ} \mathrm{C}$, las cabezas se separaron del cuerpo y se homogenizaron en Buffer fosfato de sodio-EDTA 0.1 M-1mM a pH 7.4 enfriado a $4{ }^{\circ} \mathrm{C}$, utilizando un volumen de $5.0 \mu \mathrm{L}$ por unidad, tomando $\mathrm{n}=50$ para cada ensayo. Se empleó centrifugación diferencial para extraer el citosol a $10.000 \mathrm{rpm}$ por cinco minutos y $14.0000 \mathrm{rpm}$ por diez minutos. El sobrenadante fue separado e inyectado directamente en el equipo de HPLC (Patel et al., 2013; Prasad, 2013).

\section{Análisis de Dopamina por HPLC}

El análisis se llevó a cabo en el cromatógrafo VWR Hitachi ELITE La Chromsoftware EZ-Chrom Elite ${ }^{\circledR}$ Data System, conformado con una bomba cuaternaria de 6000 psi, desgasificador en línea integrado, inyector manual con loop de $20 \mu \mathrm{L}$; columna LiChrospher ${ }^{\circledR} 100$ rp-18 endcapped ( $5 \mu \mathrm{m})$ de $125 \mathrm{~mm}$ de longitud, detector UV-VisibleHitachi L2420 a $280 \mathrm{~nm}$. La fase móvil estaba compuesta con Ácido trifluoroacétco $0.2 \%$ y Metanol (70:30 v/v) a un flujo de 0.8 $\mathrm{mL} / \mathrm{min}$. Los niveles de dopamina (DA) se calcularon mediante la ejecución de estándares y se expresaron en $\mu \mathrm{g} / \mathrm{L}$ (Prasad, 2013).

\section{Análisis estadístico}

Los resultados son presentados como la media \pm SEM para cada grupo de experimentos. Los datos fueron analizados test de normalidad D'Agostino\& Pearson y con ANOVA de una vía seguido por el post-test de Tukey para 
comparar el testigo y los grupos de tratamientos; los valores de $\mathrm{p}$ menores a 0.05 fueron considerados estadísticamente diferentes. Todos los análisis se hicieron con el software GraphPad PRISM ${ }^{\circledR}$ versión 5.0.

\section{RESULTADOS Y DISCUSIÓN |}

\section{Caracterización fitoquímica}

A través de la caracterización fitoquímica preliminar se evidenció que el extracto metanólico de $S$. ovalifolium presentó abundancia de compuestos fenólicos, y flavonoides, así como presencia de alcaloides, triterpenos, saponinas, terpenos y esteroles como se muestra en la tabla 1.

La familia Solanaceae comprende cerca de 100 géneros y 2500 especies(P. D. Cárdenas et al., 2014). Presenta propiedades antibacterial, anti-inflamatoria, antihepatotoxica, hipotensora(Gracia de Garcia et al., 1995), además de inhibir las reacciones alérgicas y la liberación de histamina (Munari et al., 2012). El género Solanum se caracteriza por su alta concentración alcaloidal y parte de su toxicidad pueden deberse a estos. Además muestran una gran variedad de metabolitos secundarios como esteroides, alcaloides, sesquiterpenos, diterpenos y antraquinonas (Wink, 2003). Los resultados obtenidos en este análisis son similares a los encontrados por (Londoño et al., 2016) para las especies Solanum arboreum y $\mathrm{S}$. ovalifoliumen donde hicieron un tamizaje fitoquímico en el que se logró detectar la presencia de estos metabolitos con la misma técnica.

\section{Caracterización de flavonoides por HPLC-DAD y espectroscopia uv-visible}

El análisis de compuestos fenólicos por HPLC se ha convertido en uno de los procedimientos analíticos más relevantes, por la simplicidad en el tratamiento de la muestra, la posibilidad de remover impurezas, permite hacer cambios en la polaridad de la fase móvil durante la corrida, los tiempos de análisis son cortos y además su reproducibilidad es alta. El quipo HPLC se acopla diferentes tipos de detectores, para estos compuestos es muy común el uso de un detector de arreglo de diodos (DAD) que permite la medición simultánea a diferentes longitudes de onda puesto que estos muestras máximos de absorción a diferentes nanómetros (Mattila et al., 2000).

Una característica que facilita el análisis de flavonoides es la presencia del anillo fenilo. El espectro ultravioleta del benceno tiene una muy buena estructura debido a la interacción vibracional y electrónica de sus niveles de energía. Este excelente cromóforo (grupo funcional que absorbe luz ultravioleta) presenta tres bandas de absorción a 184, 202 y $255 \mathrm{~nm}$. Cuando el anillo presenta sustituciones, puede por consiguiente, haber conjugaciones, las cuales van a presentar desplazamiento batocrómicos en el espectro UV y proporcionar información estructural que permita identificar sus patrones de oxidación y tipo de flavonoide(Mattila et al., 2000).

El extracto metanólico de S. ovalifoium analizado por HPLC bajo monitoreo a una longitud de onda de $280 \mathrm{~nm}$ se muestra en la Figura 2. Los tiempos de retención, las longitudes de onda que presenta cada espectro UV (bandas I y II) y tipo de flavonoide que se le atribuye de acuerdo con sus características y el espectro UV de cada pico se relacionan en la tabla 2 .

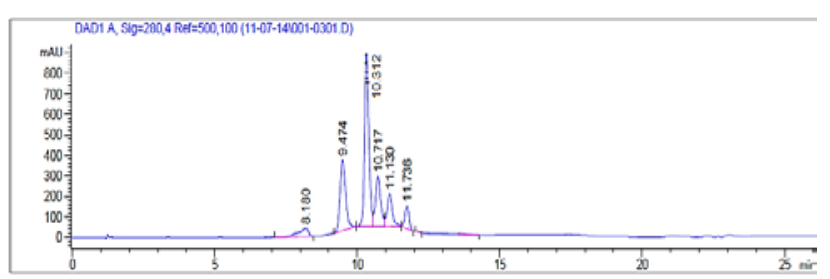

Figura 2. Cromatograma de HPLC analítico del extracto Solanum ovalifolium (UTP 99) de metanol a longitud de onda de $280 \mathrm{~nm}$. Sistema de elución gradiente $0 \%-100 \% \mathrm{MeOH}$ en ácido fórmico al $1 \%$.

A través de HPLC-DAD se pudo establecer que en el extracto metanólico de $S$. ovalifolium los tipos de flavonoides corresponden a un $16.66 \%$ de flavona-flavonol y $83.33 \%$ de dihidroflavona/dihidroflavonol.

\section{Determinación de la capacidad antioxidante}

Solanum ovalifolium mostró un buen porcentaje de actividad antioxidante del $93.56 \%$, muy cercano al control positivo Hidroquinona (96.29\%) y un valor de $I C_{50}$ de $41.5 \mu \mathrm{g} / \mathrm{mL}$. Estos resultados coinciden con otros trabajos reportados para plantas del género Solanum que han mostrado porcentajes de actividad antioxidante entre $20 \%$ y $80 \%$ y presentan valores de $\mathrm{IC}_{50}$ inferiores a $100 \mu \mathrm{g} / \mathrm{mL}$ (Braga et al., 2016; Kong et al., 2015; Valcarcel et al., 2015).

Por otro lado mediante el ensayo $\mathrm{ABTS}^{*+} \mathrm{S}$. ovalifolium presentó un porcentaje del $10.58 \%$. Y para unificar las unidades de los resultados obtenidos y comparar con otros estudios de captura de radicales libres se realizó una curva de calibración en equivalentes de Trolox para el ensayo $\operatorname{DPPH}^{\bullet}\left(y=0.950 x-1.519, R^{2}: 0.9981\right)$ y otra para el ensayo $\operatorname{ABTS}^{\circ+}\left(y=0.950 x-1.519, R^{2}\right.$ de 0.9994).

Tabla 1. Caracterización fitoquímica preliminar por cromatografía de capa delgada del extracto metanólico de Solanum ovalifolium.

\begin{tabular}{|c|c|c|c|c|c|c|c|c|c|}
\hline \multicolumn{10}{|c|}{ METABOLITOS SECUNDARIOS } \\
\hline \multirow[b]{2}{*}{ Alcaloides } & \multirow[b]{2}{*}{$\begin{array}{l}\text { Fenoles y } \\
\text { taninos }\end{array}$} & \multicolumn{3}{|c|}{ Esteroides, Triterpenos, Terpenos y Esteroles } & \multirow[b]{2}{*}{ Terpenos } & \multirow[b]{2}{*}{ Saponinas } & \multirow[b]{2}{*}{ Flavonoides } & \multirow{2}{*}{$\begin{array}{c}\text { Lactonas y } \\
\text { sesquiterpen- } \\
\text { lactonas } \\
\end{array}$} & \multirow[b]{2}{*}{ Cumarinas } \\
\hline & & Esteroides & Triterpenos & $\begin{array}{c}\text { Terpenos y } \\
\text { esteroles }\end{array}$ & & & & & \\
\hline $\begin{array}{c}+ \\
\text { Papaverina }\end{array}$ & $\begin{array}{c}++ \\
\text { Ácido tánico }\end{array}$ & ND & $\begin{array}{c}\text { ND } \\
\text { Lupeol y lanosterol }\end{array}$ & + & $\begin{array}{c}+ \\
\text { Lupeol }\end{array}$ & $\begin{array}{c}- \\
\text { Digitonina }\end{array}$ & $\begin{array}{c}++ \\
\text { Apigenina }\end{array}$ & $\begin{array}{c}\mathrm{ND} \\
\text { Digitoxina }\end{array}$ & $\begin{array}{c}\text { ND } \\
\text { Hidroxicoumarina }\end{array}$ \\
\hline
\end{tabular}

(-): Ausencia del núcleo; (+): Presencia del núcleo; (++): Presencia intermedia; (+++): Presencia abundante; ND: No determinado 
Tabla 2. Tiempo de retención de los picos obtenidos por HPLC para el extracto Solanum ovalifolium y longitudes de onda para sus respectivos espectros UV.

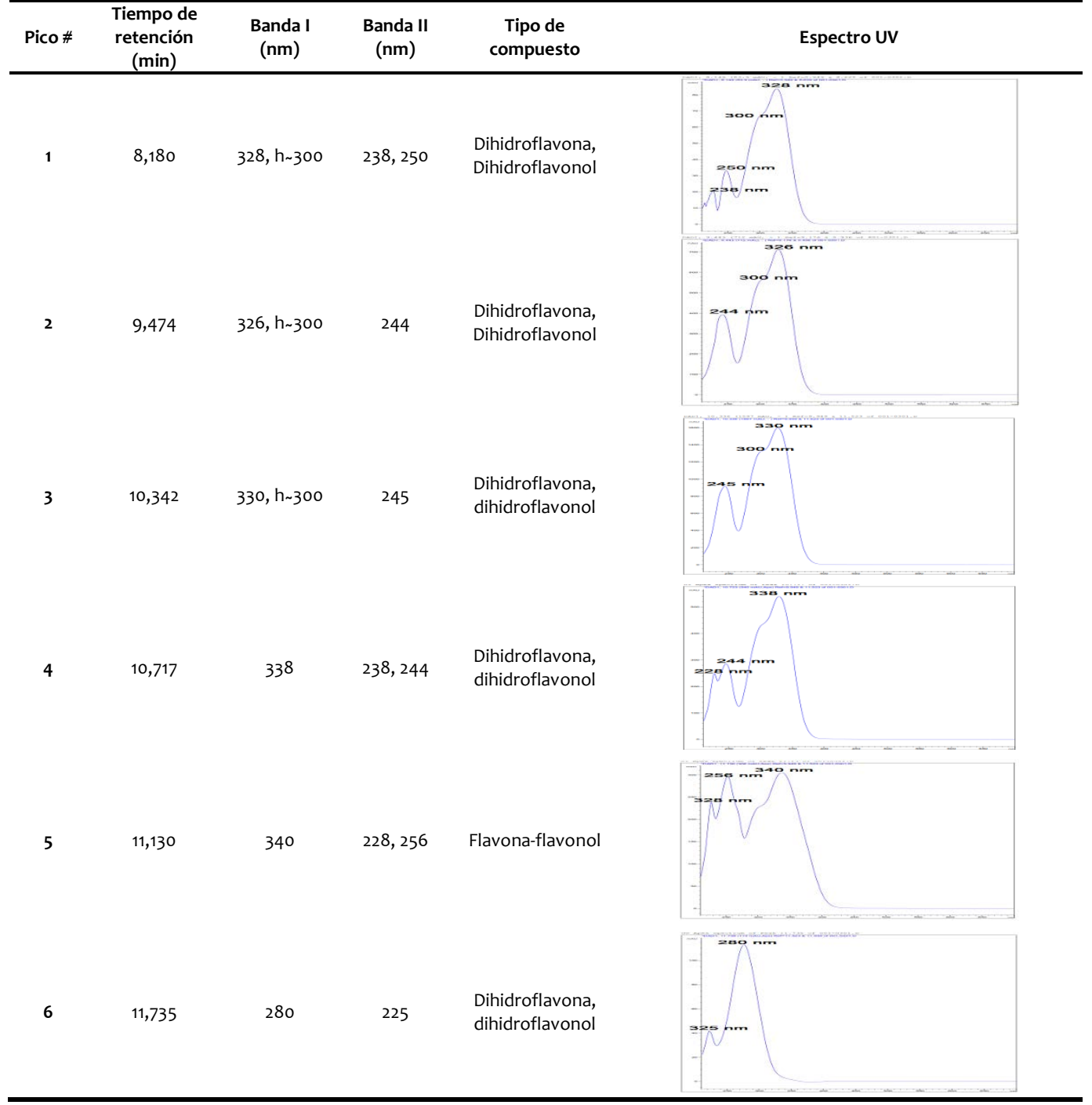

A partir de una concentración del extracto a $80 \mu \mathrm{g} / \mathrm{mL}$ se puedo determinar por el método $\mathrm{DPPH}^{{ }^{*}}$ una equivalencia de $99.99 \mathrm{mg} / \mathrm{g}$ Trolox y $\mathrm{ABTS}^{\circ+} 33.69 \mathrm{mg} / \mathrm{g}$ en peso seco Trolox. Lo anterior indica que el extracto usado a bajas concentraciones muestra buen potencial antioxidante in vitro frente a los radicales estudiados.

\section{Análisis de GSH y SOD}

Para la cuantificación de Tioles libres se realizó una curva calibración usando como tiol de referencia glutationa GSH (y = 0,0317X +0,4586, $\left.R^{2}=0.992\right)$, se obtuvo una concentración muy baja de tioles libres en Solanum ovalifolium (UTP 99) presentaron una concentración de tioles libres de 4.28 $\mathrm{GSH} / \mathrm{mL}$ extracto. De la misma manera se realizó la curva de calibración de superóxido dismutasa SOD con un coeficiente de correlación $\left(y=1,991 X+0,1585, R^{2}=0.9989\right)$, donde
Solanum ovalifolium reveló una concentración de SOD de 1.434 Unidades SOD/ mL extracto a $1000 \mathrm{ppm}$

La cantidad de enzima hallada en los extractos vegetales o moléculas miméticas a las enzimas evaluadas es relativamente baja, un resultado coherente, si se tiene presente que la funcionalidad de la enzima Superóxido dismutasa está dada por su estructura cuaternaria, donde todos los cambios en el ambiente pueden generar un daño irreversible en mayor o menor medida; modificando su estructura y en consecuencia su funcionalidad (Bindseil et al., 2001). Para la obtención del extracto, el material vegetal es sometido a diferentes cambios de temperatura y polaridades, lo que puede influir en la poca reactividad enzimática, o moléculas que puedan mimetizar las reacciones que se llevan a cabo en el proceso oxidativo por esta vía. Es preciso analizar las actividades SOD y GSH en la extracción del tejido vivo de las moscas sometidas al proceso 
de neurotoxicidad para establecer si el extracto estimula la neuroprotección o previene el estrés oxidativo.

Comportamiento y actividad locomotora de Drosophila melanogaster empleando el ensayo de geotaxis negativa

En la figura 3, se muestran los resultados del ensayo de geotaxis negativa para evaluar la concentración del extracto de S. ovalifolium a emplear como neuroprotector contra la toxicidad inducida por rotenona.

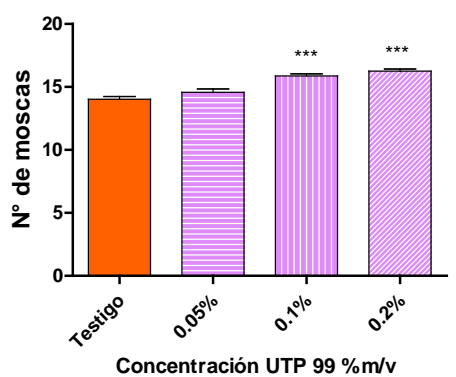

Figura 3. Geotaxis negativa de moscas macho Drosophila melanogaster expuestas a diferentes concentraciones de extracto de Solanum ovalifolium; tiempo de ascensión 6 segundos. Cada valor representa las medias de 10 experimentos independientes. Las barras de error representan los límites superior e inferior en el intervalo de confianza del $95 \%$. No se muestran diferencias significativas a $\mathrm{p}<0,05$ para la concentración de $0.1 \%$ y $0.2 \%$.

Para la elección de la concentración del extracto de Solanum ovalifolium (UTP 99) a utilizar en los ensayos del modelo in vivo, se hizo este análisis previo donde se eligió la concentración de $0,1 \%$ debido a que estimula un poco la capacidad de escape de las moscas respecto al testigo y no difiere significativamente de la concentración mayor (0,2\%). Otro factor que se debía analizar era si tenía efectos adversos o negativos sobre la capacidad locomotora, lo cual no sucedió, ya que el número de moscas que ascendieron fue mayor en todos los tratamientos respecto al testigo.

Contenido de dopamina en Drosophila melanogaster mediante análisis por HPLC-UV del efecto modulatorio del extractometanólico de $S$. ovalifolium

En la figura 4, se ve una reducción en la concentración de dopamina en las moscas expuestas a neurotoxicidad con rotenona, de esta manera los moscas pierden capacidad locomotora, sin embargo al incluir el extracto UTP 99 en la dieta de Drosophila melanogaster el neurotóxico no tiene el mismo efecto, es decir que el extracto contiene moléculas capaces de generar neuroprotección, inclusive de estimular la cantidad de dopamina producida en comparación a moscas expuestas a una dieta normal. El extracto UTP 99 aumenta la concentración de dopamina respecto al testigo e inhibe la toxicidad inducida por rotenona, dando un valor de $61.42 \mu \mathrm{g} / \mathrm{L}$ de dopamina.

En diversos estudios se destaca la capacidad antioxidante de especies de la familia Solanaceae (P. Cárdenas et al., 2015; Kumaran y Joel Karunakaran, 2007b;
Rumbaoa et al., 2009; Sabir y Rocha, 2008), se demuestra la presencia de flavonoides; flavonoles, flavonas, flavanonas, antocianidinas, catequinas y biflavonas (Prakash et al., 1995). En la familia Solanaceae se han encontrado actividades antioxidantes capaces de inhibir ulceraciones (Nguelefack et al., 2008), inflamación, tumores (Ndebia et al., 2007), entre otras.

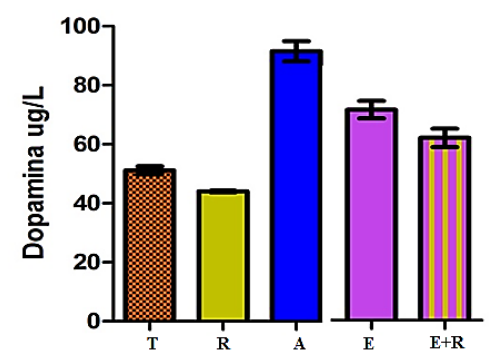

Figura 4. Análisis de Dopamina en cabezas de Drosophila melanogaster tras exposición a diferentes tratamientos. T: Alimento base, R: Alimento con rotenona a 100 uM, A: Alimento con ácido ascórbico a $0.01 \%$, E: Alimento enriquecido con extracto metanólico de S. ovalifolium a una concentración de $0.1 \%$, E+R: Tratamiento con extracto a $0.1 \%$ y rotenona a $100 \mathrm{uM}$. Las barras de error representan los límites superior e inferior en el intervalo de confianza del $95 \%$. Todos los ensayos muestran diferencias significativas a $\mathrm{p}<0,05$

En este estudio se encontró la relación de la actividad antioxidante in vitro con la capacidad de inhibir la toxicidad inducida por rotenona en Drosophila melanogaster por lo que presentó actividad neuroprotectora, lo que se notó con el modelo RING y en la cuantificación de dopamina en la región cerebral de las moscas. Es de destacar que estos son los primeros resultados publicados sobre la actividad neuroprotectora evaluada para la especie Solanum ovalifolium.

Esta propiedad podría atribuirse a la gran cantidad de compuestos fenólicos presentes en el extracto, los cuales actúan impidiendo el avance de la enfermedad de Parkinson o déficit locomotor provocado, frenando el estrés oxidativo ocasionado por los ROS producidos por la rotenona, principalmente en el complejo I de la cadena trasportadora de electrones mitocondrial en donde se da el primer fallo metabólico inicial de la mitocondria (Reducción de ATP), desde esta fase se da un mal funcionamiento de los canales iónicos de la membrana y se produce una liberación masiva de neurotransmisores y glutamato lo que genera una mayor entrada del ión $\mathrm{Ca}^{+2}$ e hiperexpresion enzimática. Las proteínas $G$ amplifican las señales inducidas por los neurotransmisores, tratando de regular la hidrolisis de fosfatidilinositol y la activación de las fosfolipasas. La producción siguiente de especies reactivas de oxígeno y nitrógeno $\left(\mathrm{O}_{2}\right.$; $\mathrm{NO}$, peroxinitrito $\left.\mathrm{y}_{2} \mathrm{O}_{2}\right)$ forma parte del proceso oxidativo celular. La peroxidación lipídica, el daño nuclear en el ADN (Dajas et al., 2003) . Los flavonoides actúan por la inhibición de muchas etapas de este proceso: Xantinooxidasa, peroxidación lipídica, 3-fosfatidilinositol quinasa, lipooxigenasa, proteínquinasa y la eliminación de radicales libres marcados en la figura 5 . 


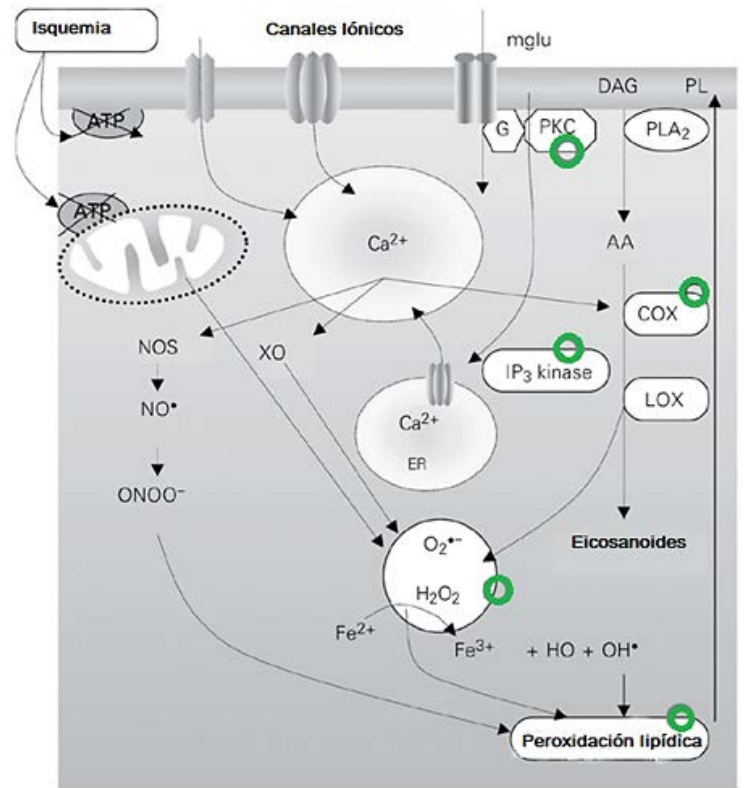

Figura 5. Proceso de Isquemia a nivel celular.(Adaptado de (Gutierrez-Merino et al., 2011). IP3Kinasa: 3 -fosfatidilinositol quinasa; AA: ácido araquidónico; COX: ciclooxigenasa; DAG: diaglicerol; PL: fosfolípidos de membrana; PLA2: fosfolipasa A2; G: proteína G; LOX: lipooxigenasa; NO: óxido nítrico; NOS: óxido nítrico sintasa; PKC: proteínquinasa C; ER: retículo endoplásmico; mglu: receptores metabotrópicos de glutamato; XO: xantino oxidasa.

Basados en la marcha fitoquímica y los resultados del perfil cromatográfico, los flavonoides de S. ovalifolium (UTP 99, Solanaceae) (16.66\% Flavona, $83.33 \%$ Dihidroflavona) podrían ser los responsables de la mayor actividad neuroprotectora, con lo que se confirma que los niveles de ROS y de peroxidación lipídica disminuyen con la administración del extracto vegetal en Drosophila melanogaster usando como neurotóxico rotenona. Existen estudios que refuerzan esta idea (Everett et al., 1996; Limón et al., 2010), donde el flavonoide más mencionado es la quercitina mostrando una capacidad antioxidante 5 veces mayor al ácido ascórbico.

Por lo tanto es necesario realizar investigaciones más profundas de aislamiento de compuestos fenólicos de Solanum ovalifolium ya que diversos autores han encontrado flavonoides que pueden inhibir la peroxidación lipídica (Kumaran y Joel Karunakaran, 2007a), con acción inflamatorias (Sabir y Rocha, 2008; Siqueira et al., 2011), moléculas que presentan antagonismo ante el ion $\mathrm{Ca}^{+2}$ (Ghisalberti, 1997), taninos con actividad anti-VIH (Sánchez-Lamar et al., 2002), flavonoides con actividad anticáncer en condiciones in vitro e in vivo (Parvathaneni et al., 2014), y flavonas con actividad nematicida.

\section{CONCLUSIONES |}

El extracto metanólico de Solanum ovalifolium contiene moléculas con actividad antioxidante in vitro frente a los radicales $\mathrm{DPPH}^{\bullet}$ y $\mathrm{ABTS}^{\bullet+}$ y neuroprotectora demostrada por los análisis con el modelo in vivo Drosophila melanogaster, estas actividades se podrían atribuir a los diferentes flavonoides encontrados en el extracto analizados por HPLCDAD. Este el primer estudio publicado en evaluar el potencial neuroprotector de la especie Solanum ovalifolium.

Con el desarrollo de este trabajo se estandarizó una metodología eficiente para evaluar la actividad neuroprotectora con el modelo de Drosophila melanogaster y la cuantificación de Dopamina por HPLC-UV.

\section{AGRADECIMIENTOS}

A la Universidad Tecnológica de Pereira, a COLCIENCIAS (Código Jl9-14-4; 5827-5822) y al Sistema General de Regalías (Código BPIN 2012000100050) por el apoyo financiero.

\section{REFERENCIAS |}

- Bindseil, K. U., Jakupovic, J., Wolf, D., Lavayre, J., Leboul, J., \& van der Pyl, D. (2001). Pure compound libraries; a new perspective for natural product based drug discovery. Drug Discovery Today, 6(16), 840-847.

- Bosson, A., Boisseau, S., Buisson, A., Savasta, M., \& Albrieux, M. (2015). Disruption of dopaminergic transmission remodels tripartite synapse morphology and astrocytic calcium activity within substantia nigra pars reticulata. Glia, 63(4), 673-683.

- Braga, P. C., Scalzo, R. L., Dal Sasso, M., Lattuada, N., Greco, V., \& Fibiani, M. (2016). Characterization and antioxidant activity of semi-purified extracts and pure delphinidin-glycosides from eggplant peel (Solanum melongena L.). Journal of Functional Foods, 20, 411-421.

- Brand-Williams, W., Cuvelier, M.-E., \& Berset, C. (1995). Use of a free radical method to evaluate antioxidant activity. LWT-Food Science and Technology, 28(1), 25-30.

- Cárdenas, P., Sonawane, P., Heinig, U., Bocobza, S., Burdman, S., \& Aharoni, A. (2015). The bitter side of the nightshades: Genomics drives discovery in Solanaceae steroidal alkaloid metabolism. Phytochemistry, 113, 24-32.

- Correa, Y. M., Mosquera, O. M., \& Nino, J. (2011). Evaluation of Colombian Rainforest plants for their DNA interaction and cytotoxic activities. International Journal of PharmTech Research, 3, 632-638.

- Coulom, H., \& Birman, S. (2004). Chronic exposure to rotenone models sporadic Parkinson's disease in Drosophila melanogaster. The Journal of Neuroscience, 24(48), 10993-10998.

- Dajas, F., Rivera-Megret, F., Abin-Carriquiry, J. A., Echeverry, C., Lafon, L., Heizen, H., . . . Morqui, A. (2003). Neuroprotection by flavonoids. Brazilian Journal of Medical and Biological Research, 36(12), 1613-1620.

- Diniz, T. C., Silva, J. C., Lima-Saraiva, S. R. G. d., \& et al. (2015). The Role of Flavonoids on Oxidative Stress in Epilepsy. Oxidative Medicine and Cellular Longevity, 2015, 9.

- Everett, S. A., Dennis, M. F., Patel, K. B., Maddix, S., Kundu, S. C., \& Willson, R. L. (1996). Scavenging of nitrogen dioxide, thiyl, and sulfonyl free radicals by the nutritional antioxidant-carotene. Journal of Biological Chemistry, 271(8), 3988-3994.

- Flagg, R. O. (1988). Carolina Drosophila Manual: Carolina Biological Supply Company Burlington.

- Gargano, J. W., Martin, I., Bhandari, P., \& Grotewiel, M. S. (2005). Rapid iterative negative geotaxis (RING): a new method for assessing age-related locomotor decline in Drosophila. Experimental Gerontology, 40(5), 386-395.

- Ghisalberti, E. (1997). Cardiovascular activity of naturally occurring lignans. Phytomedicine, 4(2), 151-166. 
- González, M. R., Hernández, R. V., del Tánago, P. G. G., LLerena, A. L., Oliver, D. P., \& León, A. C. (2016). Enfermedad de Parkinson. Panorama actual del medicamento, 40(392), 264-281.

- Gracia de Garcia, C. C., Correa, A., \& Rojas, C. (1995). Estudio fitoquímico preliminar y evaluación de la actividad antimicrobiana de algunas plantas superiores colombianas. Revista Colomiana de Ciencias Químicas Farmaceúticas (23), 42-48.

- Gutierrez-Merino, C., Lopez-Sanchez, C., Lagoa, R., K Samhan-Arias, A., Bueno, C., \& Garcia-Martinez, V. (2011). Neuroprotective actions of flavonoids. Journal of Current Medicinal Chemistry, 18(8), 1195-1212.

- Hosamani, R., \& Muralidhara. (2009). Neuroprotective efficacy of Bacopa monnieri against rotenone induced oxidative stress and neurotoxicity in Drosophila melanogaster. NeuroToxicology, 30(6), 977-985.

- Kong, Y., Huang, S., Xie, M., Xue, Y., Zou, K., \& Liu, S. (2015). The Antioxidant Activity of Scopolin from Solanum cathayanum to Nerve Cells. Medicinal Plant, 6(1), 1- 8.

- Kumaran, A., \& Joel Karunakaran, R. (2007a). In vitro antioxidant activities of methanol extracts of five Phyllanthus species from India. LWT-Food Science and Technology, 40(2), 344-352.

- Kumaran, A., \& Joel Karunakaran, R. (2007b). In vitro antioxidant activities of methanol extracts of five Phyllanthuss pecies from India. LWT-Food Science and Technology, 40(2), 344-352.

- Limón, D., Díaz, A., Mendieta, L., Luna, F., Zenteno, E., \& Guevara, J. (2010). Los flavonoides: mecanismos de acción, neuroprotección y efectos farmacológicos. Mensaje Bioquímico, 34(1), 143-155.

- Londoño, F., Cardona, W., Alzate, F., Cardona, F., elez, I. D., Upegui, Y., . . . Robledo, S. M. (2016). Antiprotozoal activity and cytotoxicity of extracts from Solanum arboreum and S. ovalifolium (Solanaceae). Journal of Medicinal Plants Research, 10(8), 100-107.

- Mattila, P., Astola, J., \& Kumpulainen, J. (2000). Determination of flavonoids in plant material by HPLC with diode-array and electro-array detections. Journal of Agricultural and Food Chemistry, 48(12), 5834-5841.

- Mosquera, O. M., Correa, Y. M., Buitrago, D. C., \& Niño, J. (2007). Antioxidant activity of twenty five plants from Colombian biodiversity. Memorias do Instituto Oswaldo Cruz, 102(5), 631-634.

- Munari, C. C., de Oliveira, P. F., de Souza Lima, I. M., de Paula Lima Martins, S., de Carvalho da Costa, J., Bastos, J. K., \& Tavares, D. C. (2012). Evaluation of cytotoxic, genotoxic and antigenotoxic potential of Solanum lycocarpum fruits glicoalkaloid extract in V79 cells. Food and Chemical Toxicology, 50(10), 3696-3701.

- Ndebia, E., Kamgang, R., \& Nkeh-ChungagAnye, B. (2007). Analgesic and anti-inflammatory properties of aqueous extract from leaves of Solanum torvum (Solanaceae). African Journal of Traditional, Complementary and Alternative Medicines, 4(2), 240-244.

- Nguelefack, T. B., Feumebo, C. B., Ateufack, G., Watcho, P., Tatsimo,et al., (2008). Anti-ulcerogenic properties of the aqueous and methanol extracts from the leaves of Solanum torvum Swartz (Solanaceae) in rats. Journal of Ethnopharmacology, 119(1), 135-140.

- Niño, J., Correa, Y., \& Mosquera, O. (2006). Antibacterial, antifungal, and cytotoxic activities of 11 Solanaceae plants from Colombian biodiversity. Pharmaceutical Biology, 44(1), 14-18.

- Niño, J., Narváez, D. M., Mosquera, O. M., \& Correa, Y. M. (2006). Antibacterial, antifungal and cytotoxic activities of eight Asteraceae and two Rubiaceae plants from Colombian biodiversity. Brazilian Journal of Microbiology, 37(4), 566-570.

- Niño Osorio, J., Morales, P. A., Correa, N., Milena, Y., Mosquera, M., Oscar, M., \& Batero, J. (2007). Extractos vegetales con actividad sobre cepas mutadas de Saccharomyces cerevisiae con deficiencia en el mecanismo de reparación del ADN. Scientia et Technica, 1(33).

- Parvathaneni, M., Battu, G. R., Gray, A. I., \& Gummalla, P. (2014). Investigation of anticancer potential of hypophyllanthin and phyllanthin against breast cancer by in vitro and in vivo methods. Asian Pacific Journal of Tropical Disease, 4, S71-S76.

- Patel, M., Vasaya, M. M., Asker, D., \& Parsons, R. B. (2013). HPLC-UV method for measuring nicotinamide $\mathrm{N}$-methyltransferase activity in biological samples: Evidence for substrate inhibition kinetics. Journal of Chromatography B, 921-922(0), 87-95.

- Prakash, A., Satyan, K., Wahi, S., \& Singh, R. (1995). Comparative hepatoprotective activity of three Phyllanthus species, $P$. urinaria, $P$. nirur and $P$. simplex, on carbon tetrachloride induced liver injury in the rat. Phytotherapy Research, 9(8), 594-596.

- Prasad, S. N. (2013). Neuroprotective efficacy of eugenol and isoeugenol in acrylamide-Induced neuropathy in rats: Behavioral and biochemical evidence. Neurochemical Research, 38(2), 330-345.

- Re, R., Pellegrini, N., Proteggente, A., Pannala, A., Yang, M., \& Rice-Evans, C. (1999). Antioxidant activity applying an improved ABTS radical cation decolorization assay. Free Radical Biology and Medicine, 26(9), 1231-1237.

- Rubin, G. M., Yandell, M. D., Wortman, J. R., Gabor, G. L., Nelson, C. R., et al., (2000). Comparative genomics of the eukaryotes. Science, 287(5461), 2204 2215 .

- Ruiz-Martínez, J., Ascacio, J., Rodríguez, R., Morales, D., \& Aguilar, C. (2011). Phytochemical screening of extracts from some Mexican plants used in traditional medicine. Journal of Medicinal Plants Research, 5(13), 2791-2797.

- Rumbaoa, R. G. O., Cornago, D. F., \& Geronimo, I. M. (2009). Phenolic content and antioxidant capacity of Philippine potato (Solanum tuberosum) tubers. Journal of Food Composition and Analysis, 22(6), 546-550.

- Sabir, S. M., \& Rocha, J. B. T. (2008). Antioxidant and hepatoprotective activity of aqueous extract of Solanum fastigiatum (false "Jurubeba") against paracetamol-induced liver damage in mice. Journal of Ethnopharmacology, 120(2), 226-232.

- Samarth, R. M., Panwar, M., Kumar, M., Soni, A., Kumar, M., \& Kumar, A. (2008). Evaluation of antioxidant and radical-scavenging activities of certain radioprotective plant extracts. Food Chemistry, 106(2), 868-873.

- Sánchez-Lamar, A., Fuentes, J. L. S., Fonseca, G., Cápiro, N., Ferrer, M., Alonzo, A., ... Llagostera, M. (2002). Assessment of the potential genotoxic risk of Phyllantus orbicularis HBK aqueous extract using in vitro and in vivo assays. Toxicology Letters, 136(2), 87-96.

- Siqueira, S., Falcão-Silva, V. d. S., Agra, M. d. F., Dariva, C., Siqueira-Júnior, J. P. d., \& Fonseca, M. J. V. (2011). Biological activities of Solanum paludosum Moric. extracts obtained by maceration and supercritical fluid extraction. The Journal of Supercritical Fluids, 58(3), 391-397.

- Uversky, V. N. (2004). Neurotoxicant-induced animal models of Parkinson's disease: understanding the role of rotenone, maneb and paraquat in neurodegeneration. Cell and Tissue Research, 318(1), 225-241.

- Valcarcel, J., Reilly, K., Gaffney, M., \& O’Brien, N. M. (2015). Antioxidant activity, total phenolic and total flavonoid content in sixty varieties of potato (Solanum tuberosum L.) grown in Ireland. Potato Research, 58(3), 221244.

- Veličković, D., Stankov-Jovanović, V., Mitić, V., Kostić, D., \& Palić, R. (2013). Antimicrobial and antioxidant activities of essential oil and crude extracts of Hypericum tetrapterum Fries (Hypericaceae). Journal of Medicinal Plants Research, 4(14), 1441-1445.

- Wagner, H., \& Bladt, S. (1996). Plant drug analysis: a thin layer chromatography atlas: Springer Science \& Business Media.

- Wink, M. (2003). Evolution of secondary metabolites from an ecological and molecular phylogenetic perspective. Phytochemistry, 64(1), 3-19.

- Yang, W.-M., Liu, J.-K., Qin, X.-D., Wu, W.-L., \& Chen, Z.-H. (2004) Antioxidant activities of three dihydrochalcone glucosides from leaves of Lithocarpus pachyphyllus. Zeitschrift für Naturforschung. C, Journal of Biosciences, 481-484. 